\title{
Cytomegalovirus ileocolitis in a rheumatoid arthritis patient: case report and literature review
}

\author{
M.S. Dag, I.H. Turkbeyler, Z.A. Ozturk, B. Kısacık, E. Tutar, A. Kadayıfçı \\ Department of Internal Medicine, School of Medicine, Gaziantep University, Turkey
}

\begin{abstract}
SUMMARY
Rheumatoid arthritis (RA) is an autoimmune, systemic, chronic, inflammatory disease generally treated with various immunosuppressive drugs. Cytomegalovirus (CMV) is an opportunistic, viral infection that is commonly seen in immunosuppressed patients. A sixty-four-year old female diagnosed with RA and treated with immunosuppressive agents was admitted to our rheumatology outpatient service with complaints of diarrhea and abdominal pain, which had lasted longer than four weeks. The patient's colonoscopy revealed inflamed and ulcerated areas in the colon and in the terminal ileum. A biopsy showed intra-nuclear inclusion particles consistent with CMV. We started an oral valganciclovir therapy in this serum-CMV-polymerase chain reactionpositive patient. The concomitant use of immunosuppressive agents and anti-viral drugs eased the patient's complaints, and the endoscopic picture improved. Consequently, cytomegalovirus ileocolitis in immunosuppressed patients admitted with severe diarrhea must be considered in the differential diagnosis.
\end{abstract}

Key words: Rheumatoid arthritis, Cytomegalovirus, Ileocolitis.

\section{INTRODUCTION}

$\mathrm{R}$ heumatoid arthritis (RA) is an autoimmune, systemic, chronic, inflammatory disease characterized by peripheral joint involvement. It often leads to deformity and is treated with various immunosuppressive drugs (1). Cytomegalovirus (CMV), a member of the beta-herpesvirus family, causes an infectious condition which may lead more frequently to morbidity and mortality in immunosuppressed patients $(2,3)$. Contrary to what is commonly believed, it may also lead to serious and lethal infectious conditions in immunocompetent patients (4).

It may involve any part of the gastrointestinal system, but it affects more frequently the colon $(4,5)$. This disease generally starts as an opportunistic infection, hence its onset and severity are very difficult to predict. RA and CMV rarely coexist in colitis patients $(6,7)$. This study reports a case of a patient under immunosuppressive therapy with cytomegalovirus-associated ileocolitis, who was successfully treated with anti-viral drugs. This treatment was administered without discontinuing the immunosuppressive agents.

\section{CASE REPORT}

A 64-year old female diagnosed with RA, who was receiving immunosuppressive agents, was admitted to our rheumatology outpatient service with complaints of diarrhea (10-15 times/day), and abdominal pain that had lasted for longer than four weeks. The patient's history revealed that she had been diagnosed with RA and hypertension 5 years before. She had received $5 \mathrm{mg} /$ day of prednisolone, $10 \mathrm{mg}$ /day of leflunomide, $15 \mathrm{mg} /$ week of methotrexate for 2 years, and also metronidasol as antidiarrhea treatment for two weeks. However, symptoms had not improved.

The patient's physical examination did not reveal any specific disorder, except for a minimal, diffuse, abdominal discomfort, and limited hand deformity. Laboratory markers were: white blood cells $5,100 / \mu \mathrm{L}$,
Corresponding author: Ibrahim Halil Türkbeyler Department of Internal Medicine School of Medicine

Gaziantep University, Turkey E-mail: turkbeyler@mynet.com 
hemoglobin $11.3 \mathrm{~g} / \mathrm{dL}$, platelets $268,000 /$ $\mu \mathrm{L}$, erythrocyte sedimentation rate 26 $\mathrm{mm} / \mathrm{h}$, and C-reactive protein $2.9 \mathrm{~g} / \mathrm{dL}$. The patient's stool sample was examined with macroscopic cultures and submitted to a microscopic evaluation, but it showed no significant positive findings. Amoeba, Giardia, and Clostridium difficile toxins A and B were negative. The patient's colonoscopy revealed mucosal inflammation, erosions, and superficial ulcers in the terminal

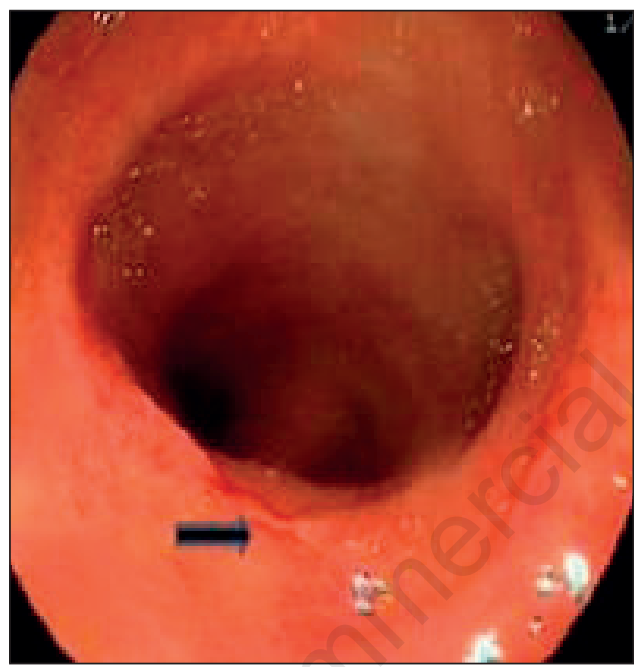

Figure 1 - Endoscopic evidence of mucosal inflammation, erosions, and superficial ulcers in terminal ileum.

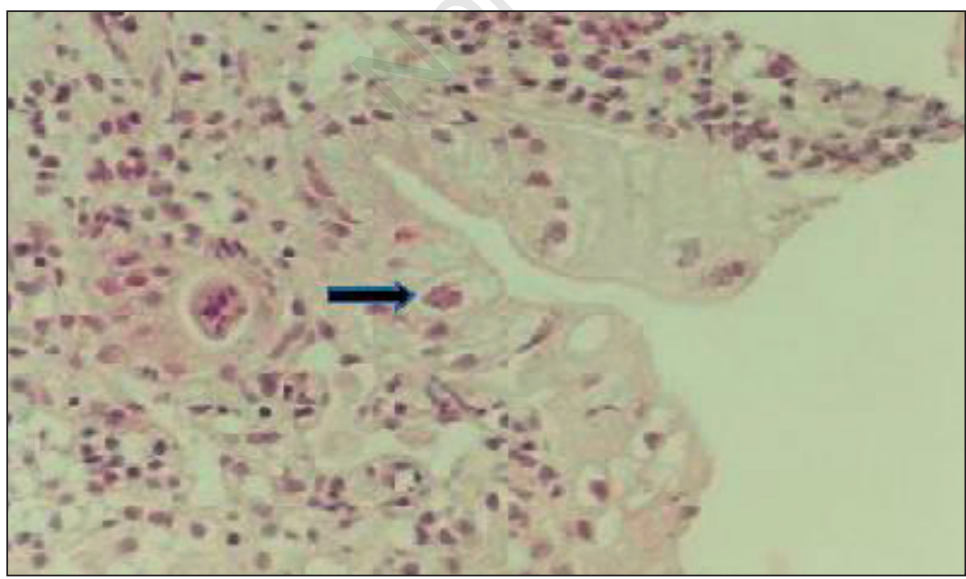

Figure 2 - Histopathologic evaluation of the terminal ileum and colon tissue samples has shown intra-nuclear inclusion bodies in epithelial cells of the enlarged surface. These were consistent with an increase in inflammatory cells and cytomegalovirus infection (hematoxilyn \& eosin staining, X100). ileum, and also edema, hyperemia, and a granular appearance in the descending colon and transverse colon mucosa (Fig. 1). The histopathological evaluation of the terminal ileum and colon tissue samples showed intra-nuclear inclusion bodies in the epithelial cells of the enlarged surface; this was consistent with an increase in inflammatory cells and CMV infection (Fig. 2). We started valganciclovir (450 mg twice a day). The patient had a polymerase chain reaction (PCR) with 2,855 copies/mL. On the fifth day of treatment, the patient's diarrhea symptoms improved significantly, and on the seventh day were almost completely relieved. After two weeks, this treatment was extended for one more week. Before and after the CMV therapy, the patient's DAS 28 score was less than 2.6. The patient received a follow-up treatment with immunosuppressive agents, and her complaints did not reappear.

\section{DISCUSSION AND CONCLUSIONS}

CMV seropositivity has been reported in around $40-100 \%$ of the population in developed and developing countries (2). In most immunosuppressed patients, CMV was observed in the reactivated form, while it is mostly a primary infection in immunocompetent patients (2). In our case, we detected a CMV-associated ileocolitis in an immunosuppressed RA patient.

Despite some rare cases of RA patients with CMV colitis, no ileitis or ileocolitis had ever been reported before (6-8). A recent case report describes a patient who was receiving rituximab and methotrexate and was diagnosed with this disorder by rectosigmoidoscopy along with serum PCR positivity. She was first treated with ganciclovir for seven days, then switched to valganciclovir for 15 days, and her complaints almost disappeared (6). Another patient was diagnosed by means of a histopathologic and immunohistochemical analysis of biopsy specimens; this patient's symptoms eased without any anti-viral therapy (7). However we could not find any 
further details about this case. Our patient was diagnosed through both a histopathologic evaluation of biopsy specimens, and positive laboratory markers. The patient responded rapidly to valganciclovir $(450 \mathrm{mg}$ twice-a-day).

Excluding RA, there are other case reports of CMV-associated ileitis. Some were treated with an immunosuppressive therapy, others were associated with an immunodeficiency. In an AIDS case, a CMVassociated ileal perforation was reported as a cause of mortality (3). A patient with Crohn's disease, who had an ileocolonic involvement and received an immunosuppressive therapy, was affected by a CMV ileitis. He was subsequently treated with valganciclovir (9). Another immunosuppressed patient diagnosed with lupus nephritis developed a CMV-associated terminal ileitis and was treated with ganciclovir (10). These complications were observed in a liver-transplanted patient who was receiving an immunosuppressive therapy. This patient was diagnosed with CMV ileitis and started ganciclovir and foscarnet sodium; however, his complaints did not improve, and surgery was required to treat fibrosis (11). The literature reports that another rare gastrointestinal disorder related to CMV is esophagitis. Akin et al. described 3 giant-cell arthritis patients who were treated with an immunosuppressive therapy. These patients had taken methyl prednisolone, and recovered after a treatment with intravenous ganciclovir (12). Gastrointestinal involvement in CMV can be associated with fever, abdominal pain, nausea, vomiting, and bloody or watery diarrhea (3-6, 9). For diagnostic purposes, radiologic, endoscopic, histopathological, and serological methods can be used; however, the primary condition for this diagnosis is to suspect the presence of a CMV infection (4). The presence of intranuclear inclusion particles with enlarged cells, in the histopathologic and immunohistochemical analyses is very important $(2,4,7,9,10)$. In our case, the predominant symptoms of the patient were watery diarrhea and abdominal pain; she was diagnosed by detecting inclusion particles in enlarged cells, along with CMV PCR positivity. Due to some technical limitations, we could not conduct immunohistochemical staining.

Although immunosuppressed patients with CMV-associated colitis or ileitis were generally treated with anti-viral therapies, different approaches were used to treat immunocompetent patients $(3-7,9,10,13)$. There is no established guideline that state when an anti-viral therapy is required. It is definitely inevitable in cases where patients are suffering from massive bleeding, intestinal obstruction, perforation, or organ transplantation, due to long-lasting immunosuppressive therapy (3, 9-11). In a recent study of 44 patients with CMV colitis (age over 55, male, with comorbidities), conditions requiring surgery were regarded as negative survival factors. In particular, $31.8 \%$ of total cases showed a spontaneous improvement with a nonspecific therapy (5). Rafailidis et al. described 89 immunocompetent patients and concluded that a life-threatening CMV infection was not rare (4). In all cases reported in the literature the immunosuppressive therapy has been stopped. As infection is drug-dependent, it is appropriate to discontinue the immunosuppressive therapy in these patients. Considering these findings, the initiation of an anti-viral therapy needs to be evaluated on a case-by-case basis. Despite the widespread use of ganciclovir, also valganciclovir combined with foscarnet sodium can still be used in resistant and severe cases $(4,6,9,10)$. The treatment lasts on average 2-4 weeks, and in this case, the patient was treated with valganciclovir. After the 3-week treatment, an improvement in the clinical, serological, and endoscopic pictures was observed.

Leflunomide is an immunomodulatory drug that may inhibit mitochondrial enzyme dihydro-orotate dehydrogenase, the proliferation of autoimmune $T$ cells and the production of autoantibodies by B cells. However leflunomide also causes a very low increase in the proportion of infections (14). Methotrexate inhibits dihydrofolate reductase and other folate-dependent enzymes. The main effect of methotrexate 
is the inhibition of thymidylate and purine synthesis in patients with rheumatoid arthritis and has shown to slightly increase the infection rate (15). Glucocorticoids have profound effects on the cellular functions of leukocytes and endothelial cells, resulting in a reduced ability of leukocytes to adhere to the vascular endothelium and leave the bloodstream. The entry to the sites of infection and tissue injury is impaired, resulting in the suppression of the inflammatory response. The reduction in endothelial adhesion may be due to the direct effects of glucocorticoids on the expression of adhesion molecules on both leukocytes and endothelial cells, as well as indirect effects due to the inhibitory effects of glucocorticoids on the transcription of cytokines, such as interleukin-1 (IL-1) or tumor necrosis factor (TNF), that upregulate the expression of endothelial adhesion molecules. The systemic glucocorticoid therapy is associated with a dose-dependent increase in the risk of infection, especially with common bacterial, viral, and fungal pathogens $(16,17)$. In the literature, tumor necrosis factor $\alpha$ is reported to play a key role in viral infections. A large number of TNF- $\alpha$ blocker-related CMV infections has also been reported (18). Besides taking methotrexate and leflunomide, our patient was administered a steroid therapy which is known to reduce the TNF- $\alpha$ level and in particular to increase the risk of CMV colitis. As a result of this investigation, the diagnosis needs to be reviewed in the presence of cytomegalovirus ileocolitis in immunosuppressed patients diagnosed with RA, or other diseases with severe diarrhea.

\section{REFERENCES}

1. Martin L. Rheumatoid arthritis: symptoms, diagnosis, and management. Nurs Times. 2004; 100: 40-4.

2. De la Hoz RE, Stephens G, Sherlock C. Diagnosis and treatment approaches to CMV infections in adult patients. J Clin Virol. 2002; 25: 1-12.

3. Meza AD, Bin-Sagheer S, Zuckerman MJ, Morales CA, Verghese A. Ileal perforation due to cytomegalovirus infection. J Natl Med Assoc. 1994; 86: 145-8.

4. Rafailidis PI, Mourtzoukou EG, Varbobitis IC,
Falagas ME. Severe cytomegalovirus infection in apparently immunocompetent patients: a systematic review. Virol J. 2008; 5: 47.

5. Galiatsatos P, Shrier I, Lamoureux E, Szilagyi A. Meta-analysis of outcome of cytomegalovirus colitis in immunocompetent hosts. Dig Dis Sci. 2005; 50: 609-16.

6. Vallet H, Houitte R, Azria A, Mariette X. Cytomegalovirus colitis and hypo-IgG after rituximab therapy for rheumatoid arthritis. J Rheumatol. 2011; 38: 965-6.

7. Kazumi E. A case of cytomegalovirus colitis associated with rheumatoid arthritis. Nagasaki Med J. 2003; 78: 1-6.

8. Taillandier J, Alemanni M, Manigand G. A case of cytomegalovirus colitis in rheumatoid polyarthritis. Rev Rhum Mal Osteoartic. 1991; 58: 638-9.

9. Le ST, Lee SS, Prideaux L, Block LA, Moore GT. Primary cytomegalovirus ileitis complicated by massive gastrointestinal haemorrhage in a patient with steroid refractory Crohn's disease. Intern Med J. 2010; 40: 788-91.

10. Khan FN, Prasad V, Klein MD. Cytomegalovirus enteritis mimicking Crohn's disease in a lupus nephritis patient: a case report. World J Gastroenterol. 2009; 15: 4327-30.

11. Navaneethan U, Venkatesh PG, Wang J. Cytomegalovirus ileitis in a patient after liver transplantation-differentiating from de novo IBD. J Crohns Colitis. 2011; 5: 354-9.

12. Akın S, Tufan F, Bahat G, Saka B, Erten N, Karan MA. Cytomegalovirus esophagitis precipitated with immunosuppression in elderly giant cell arteritis patients. Aging Clin Exp Res. 2013; 25: 215-8. Erratum in: Aging Clin Exp Res. 2013; 25: 219.

13. Momin N, Telisinghe PU, Chong VH. Cytomegalovirus colitis in immunocompetent patients. Singapore Med J. 2011; 52: 170-2.

14. Fox RI, Herrmann ML, Frangou CG, Wahl GM, Morris RE, Kirschbaum BJ. How does leflunomide modulate the immune response in rheumatoid arthritis?. Bio Drugs. 1999; 12: 301-15.

15. McLean-Tooke A, Aldridge C, Waugh S, Spickett GP, Kay L. Methotrexate, rheumatoid arthritis and infection risk: what is the evidence? Rheumatology (Oxford). 2009; 48: 867-71.

16. Fauci AS, Dale DC, Balow JE. Glucocorticosteroid therapy: mechanisms of action and clinical considerations. Ann Intern Med. 1976; 84: 304

17. Sakuma Y, Katoh T, Owada K, et al. Initial functional status predicts infections during steroid therapy for renal diseases. Clin Nephrol. 2005; 63: 68.

18. Sari I, Birlik M, Gonen C, Akar S, Gurel D, Onen F, et al. Cytomegalovirus colitis in a patient with Behcet's disease receiving tumor necrosis factor alpha inhibitory treatment. World J Gastroenterol. 2008; 14: 2912-4. 